\section{Quadhelix: una herramienta en el manejo del paciente con secuela de fisura labio-alveolo-palatina. Reporte de tres casos}

\section{Quadhelix: a tool for cleft lip and palate patients management. Report of three cases}

\section{Caso Clínico}

Lucia Lozano Llaury 1, a Alejandra Altamirano Pacheco 1, a Jessica Lenkey Ramos ${ }^{1, a}$, Jennifer Saavedra Vargas ${ }^{1, a}$, Leslie Gil Mori 1, a, Elmer Flores Leiva 1, b

${ }^{1}$ Universidad Nacional Mayor de San Marcos. Lima, Perú. a Cirujano Dentista.

${ }^{b}$ Especialista en Ortodoncia y Ortopedia Maxilar.

\section{Correspondencia:}

Lozano Llaury Lucia: lucial_95@hotmail.com

Jr. Fray Luis de León 402. San Borja - Lima ORCID: 0000-0003-4112-1469

\section{Coautores:}

Alejandra Altamirano Pacheco: ale_129_7@hotmail.com Jessica Lenkey Ramos: jessilenkey@hotmail.com Jennifer Saavedra Vargas: jennsaavedrav@gmail.com Leslie Gil Mori: lesky_10@hotmail.com

Elmer Flores Leiva: eflole05@yahoo.com

\section{Editora:}

Rosse Mary Falcón-Antenucci

Universidad Inca Garcilaso de la Vega, Lima, Perú.

Conflicto de intereses: sin conflicto de intereses.

Fuente de financiamiento: autofinanciado.

Recibido: 19/10/19

Aceptado: $14 / 02 / 20$

\section{Resumen}

Aceptado: $14 / 02 / 20$

Las fisura labio-alveolo-palatina son las anomalías congénitas más frecuentes, por lo que el ortodoncista debe conocer el manejo transversal en estos pacientes, su omisión puede hacer que se agrave la fisura, porque los efectos biomecánicos en estos pacientes merecen especial cuidado debido a su estructura maxilar. La serie de casos incluye tres pacientes, uno con secuela de fisura labio-alveolo-palatina unilateral derecho, el otro bilateral y el último unilateral izquierdo. La constricción transversal maxilar y la discrepancia en relación con el ancho mandibular, llevó a la elección del uso del quadhelix para resolver este problema. En los tres casos se lograron los objetivos transversales del tratamiento. Se concluyó que, el quadhelix representa una alternativa razonable teniendo como ventajas ser un dispositivo de expansión lenta que produce menos resistencia tisular alrededor de las estructuras circunmaxilares y, mejora la formación de hueso en la sutura intermaxilar; de igual manera al tener mayores efectos dentoalveolares, no produce pérdida ósea a nivel vestibular; adicionalmente presenta una mejor adaptación muscular y bajas probabilidades de recidiva.

Palabras clave: Técnica de expansión palatina; Fisura del paladar; Aparatos ortodóncicos (fuente DeCS BIREME).

\begin{abstract}
As cleft lip and palate is one of the most frequent congenital anomalies, orthodontists should know about transverse management in these patients, to ignore it might aggravate the fissure, as the biomechanical effects in these patients require special care due to their maxillary structure. This case series include three patients, one with a sequel of right unilateral cleft lip and palate, one bilateral and the last one with left unilateral cleft lip and palate. The maxillary transverse constriction and the discrepancy with the mandibular width, led to choice quadhelix appliance to solve this problem. In all three cases the transversal treatment objectives were achieved. In conclusion the quadhelix appliance is a reliable alternative which has advantages such as a slow expansion producing less tissue resistance around the circummaxillary structures and improves bone formation in the intermaxillary suture; similarly, it has greater dentoalveolar effects that does not
\end{abstract}


cause buccal cortical bone loss; additionally, it shows better muscular adaptation and low chance of relapse.

Keywords: Palatal expansion technique; Cleft palate; Orthodontic appliances (source: MeSH NLM).

\section{Introducción}

Las fisuras labio palatinas (FLAP) se definen como una malformación congénita de diversos grados de severidad, que abarca desde una simple depresión hasta una fisura de grosor completo del labio, juntamente con el alveolo y el paladar ${ }^{1}$. Las FLAP representan un alto porcentaje de todas las malformaciones congénitas y constituyen la anomalía congénita craneofacial más frecuente ${ }^{2}$.

Generalmente los pacientes con FLAP son sometidos a cirugías de reparación de labio durante los primeros ańos de vida, seguidas de la reparación del paladar duro y blando; estas cirugías traen como consecuencia, que el crecimiento y el desarrollo de los segmentos maxilares se vean comprometidos por los tejidos cicatriciales ${ }^{3}$.

En pacientes con paladar fisurado completo bilateral (BCLP en sus siglas en inglés) se ha demostrado que los segmentos maxilares rotan hacia medial, con fulcrum localizado en la tuberosidad maxilar determinando así una constricción progresiva anterior. Por lo tanto, la distancia intercanina muestra una mayor reducción en comparación con el ancho intermolar en este tipo de pacientes ${ }^{4} ; \mathrm{y}$ al mismo tiempo, se produce una reducción significativa del overjet, debido a la retrusión de la premaxila ${ }^{5}$. Incluso en los pacientes no operados, los pacientes alcanzan el período de dentición mixta con una constricción severa anterior versus un ancho maxilar posterior normal o casi normal ${ }^{6}$.

El ortodoncista debe hacer un manejo transversal y sagital, considerando evitar agravar la fisura. Siendo el manejo transversal el procedimiento más complejo ${ }^{7}$, porque los efectos biomecánicos en estos pacientes son diferentes, debido a su estructura maxilar especial ${ }^{8,9}$.

Para la expansión maxilar en los niños con FLAP, hay dos opciones terapéuticas disponibles: expansión rápida del maxilar (ERM) con aparatos tipo Hyrax o Haas, y expansión lenta del maxilar (ELM) con dispositivo de quadhelix ${ }^{5,10}$.

Los procedimientos de ELM producen menos resistencia tisular alrededor de las estructuras circunmaxilares y, por lo tanto, mejoran la formación de hueso en la sutura intermaxilar, lo que teóricamente debería eliminar o reducir la limitación de la ERM ${ }^{11,12}$. Por otro lado, la expansión lenta del maxilar tiene más efectos dentoalveolares con pequeñas repercusiones en la base maxilar, y no hay pérdida ósea a nivel vestibular en comparación con la expansión rápida ${ }^{13}$. Además, también tiene ventajas como mejor adaptación muscular y bajo riesgo de recidiva ${ }^{12}$.

Por otro lado, el dispositivo de quadhelix como lo describió Ricketts ${ }^{14}$ en 1975 o el dispositivo "W" anterior fueron utilizados con gran éxito en el tratamiento temprano de aquellos pacientes con paladar fisurado en los que los segmentos maxilares laterales se habían colapsado detrás de los procesos premaxilares sobresalientes 10,15. Los efectos incluyen la desrotación de los molares superiores, así como cierta expansión de los procesos maxilares laterales ${ }^{15}$.

Durante la expansión es importante preparar el maxilar para que reciba el injerto óseo secundario ${ }^{16}$. Sin embargo, no existe un protocolo universal para la expansión maxilar antes de éste ${ }^{17}$. En pacientes con BCLP, la corrección de la constricción maxilar generalmente se realiza antes del procedimiento de injerto óseo alveolar secundario ${ }^{14}$. El alineamiento de los segmentos, alcanzados con éxito con el quadhelix, provee paredes laterales para la realización del injerto óseo alveolar ${ }^{16}$.

La expansión palatina previa al injerto de hueso alveolar compromete un periodo relativamente corto de tratamiento con aparatología fija apuntando a lograr una forma de arco óptima que mejore el acceso para la cirugía. El injerto óseo es usualmente emprendido cuando el canino permanente del lado de la fisura muestra entre la mitad a dos tercios de desarrollo radicular. La expansión pre-injerto óseo, inicia aproximadamente 12 meses antes de la cirugía y es completada usando aparatología fija superior; esta aparatología debe mantenerse de 4-6 meses post-injerto, para estabilidad ${ }^{18}$.

Los que están a favor de la expansión seguida del injerto citan las ventajas de que la consolidación ósea es mejorada cuando el injerto es colocado bajo una carga dinámica durante la cicatrización y que hay un defecto de tejido blando más pequeño para cerrar, menor dificultad para obtener un volumen óseo adecuado y un defecto más estrecho, que regenerará el hueso más rápidamente. El injerto óseo debe ser cargado con fuerzas ortodónticas leves para brindar una estimulación fisiológica ${ }^{19}$.

Después de todo lo mencionado anteriormente y viendo la necesidad de conocer el manejo transversal del maxilar en este tipo de pacientes, presentamos esta serie de casos que incluye tres pacientes, uno con secuela de FLAP unilateral derecho, el otro bilateral y el último unilateral izquierdo en los cuales se optó por el uso del quadhelix para resolver los problemas transversales.

\section{Reporte de casos}

\section{Caso 1}

JMVG paciente masculino de 11 años y 7 meses de edad, con secuela de labio y paladar fisurado unilateral derecho, sin presencia de injerto alveolar. 
Dentro de las características faciales es un paciente mesofacial, perfil cóncavo, con dentición mixta y crecimiento remanente, tercio inferior disminuido, asimetría facial vertical, retrusión del labio superior y mentón protruído (Figura. 1).

A la evaluación intraoral presenta una maloclusión Clase III de Angle, múltiples piezas retenidas, overjet -2 $\mathrm{mm}$, overbite $62 \%$, distancia intercanina superior de 23 $\mathrm{mm}$ e inferior de $25 \mathrm{~mm}$, distancia intermolar superior de $43 \mathrm{~mm}$ e inferior de $39 \mathrm{~mm}$ (Figura 2).

En la radiografía panorámica se evidencian las piezas retenidas y la presencia de la fisura en el cuadrante superior derecho. En la radiografía lateral se evidencia la relación esquelética Clase III y perfil óseo cóncavo. Dentro de los exámenes auxiliares también se incluyó una tomografía cone beam (Figura 3)
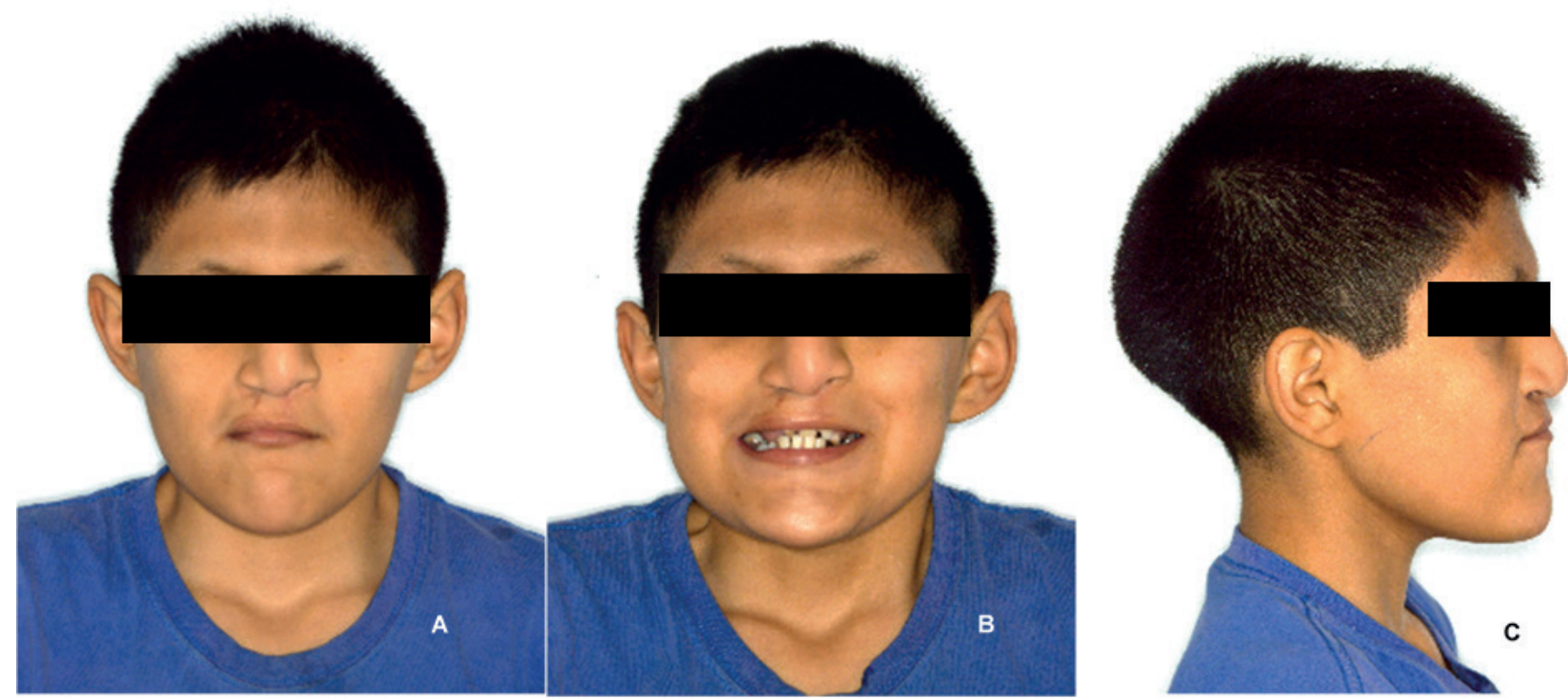

Figura 1. Fotografías extraorales iniciales del caso 1. A. Fotografía frontal. B. Fotografía de sonrisa. C. Fotografía de perfil

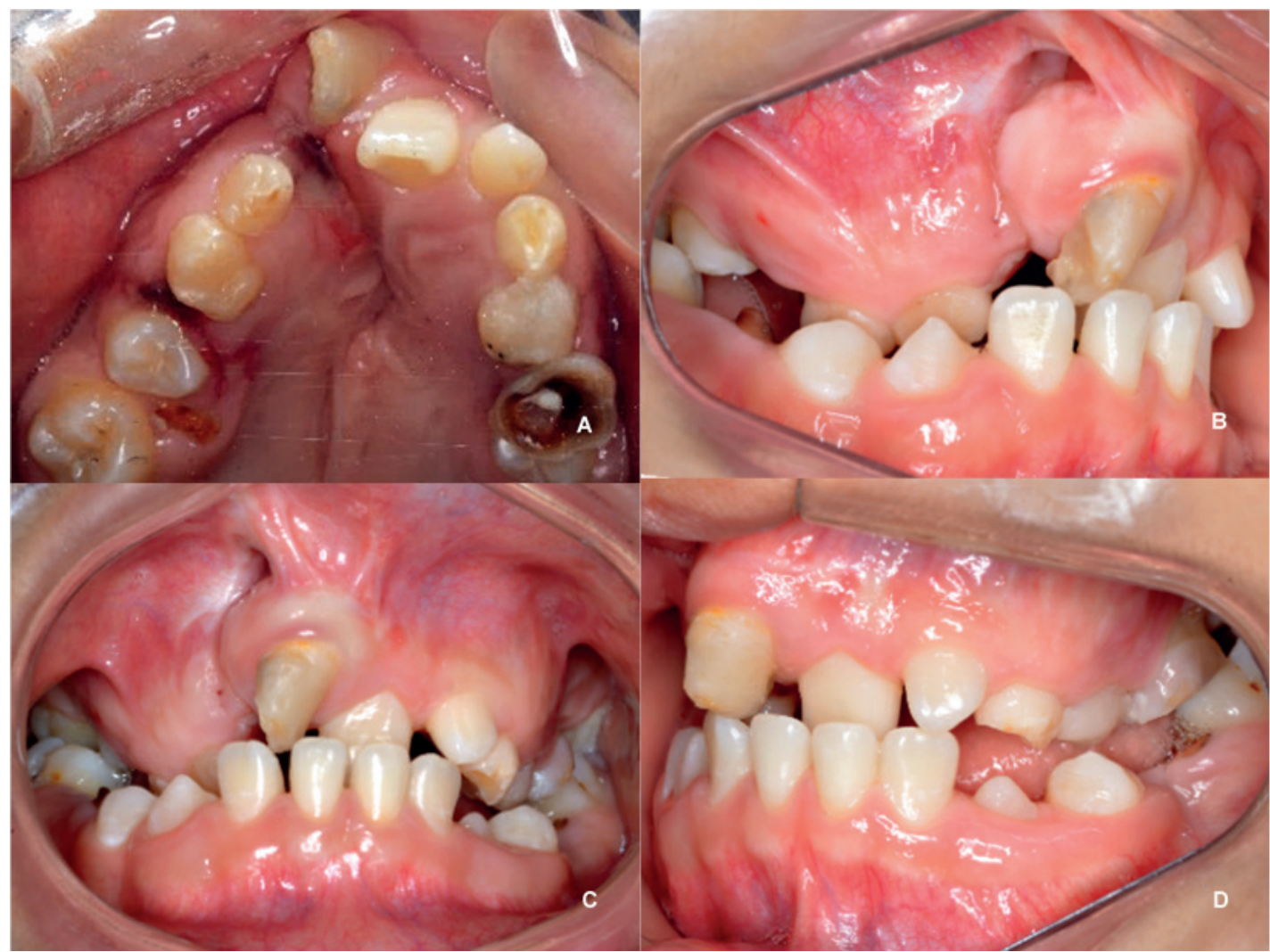

Figura 2. Fotografías intraorales iniciales del caso 1. A. Fotografía oclusal. B. Fotografía lateral derecha. C. Fotografía frontal. D. Fotografía lateral izquierda 
El plan de tratamiento consistió en la instalación de un expansor quadhelix para arcada superior y arco lingual fijo para arcada inferior. Luego de 4 activaciones en 11 meses se logró una distancia intermolar de $48 \mathrm{~mm}$ y una distancia intercanina de $29 \mathrm{~mm}$, mejorando la forma de la arcada superior y la mordida cruzada posterior (Figura 4). Luego se instaló arco de Connecticut con componente protrusivo para tratar de corregir el problema anteroposterior. Paciente actualmente continúa en tratamiento y aún no se ha realizado el injerto alveolar.

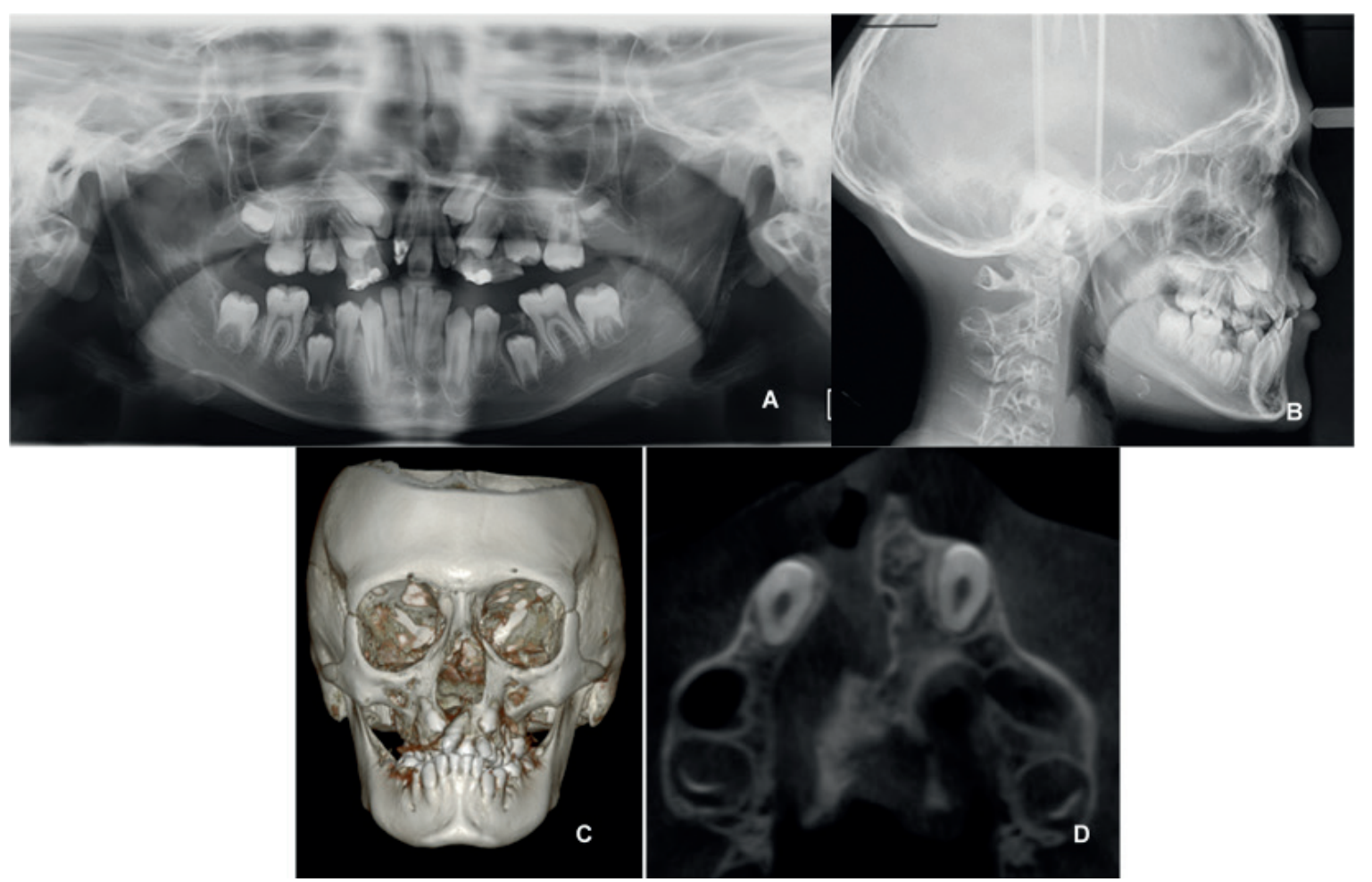

Figura 3. Exámenes auxiliares iniciales del caso 1. A. Radiografía panorámica. B. Radiografía cefalométrica. C. Vista 3D de la tomografía cone beam. D. Corte axial de tomografía cone beam

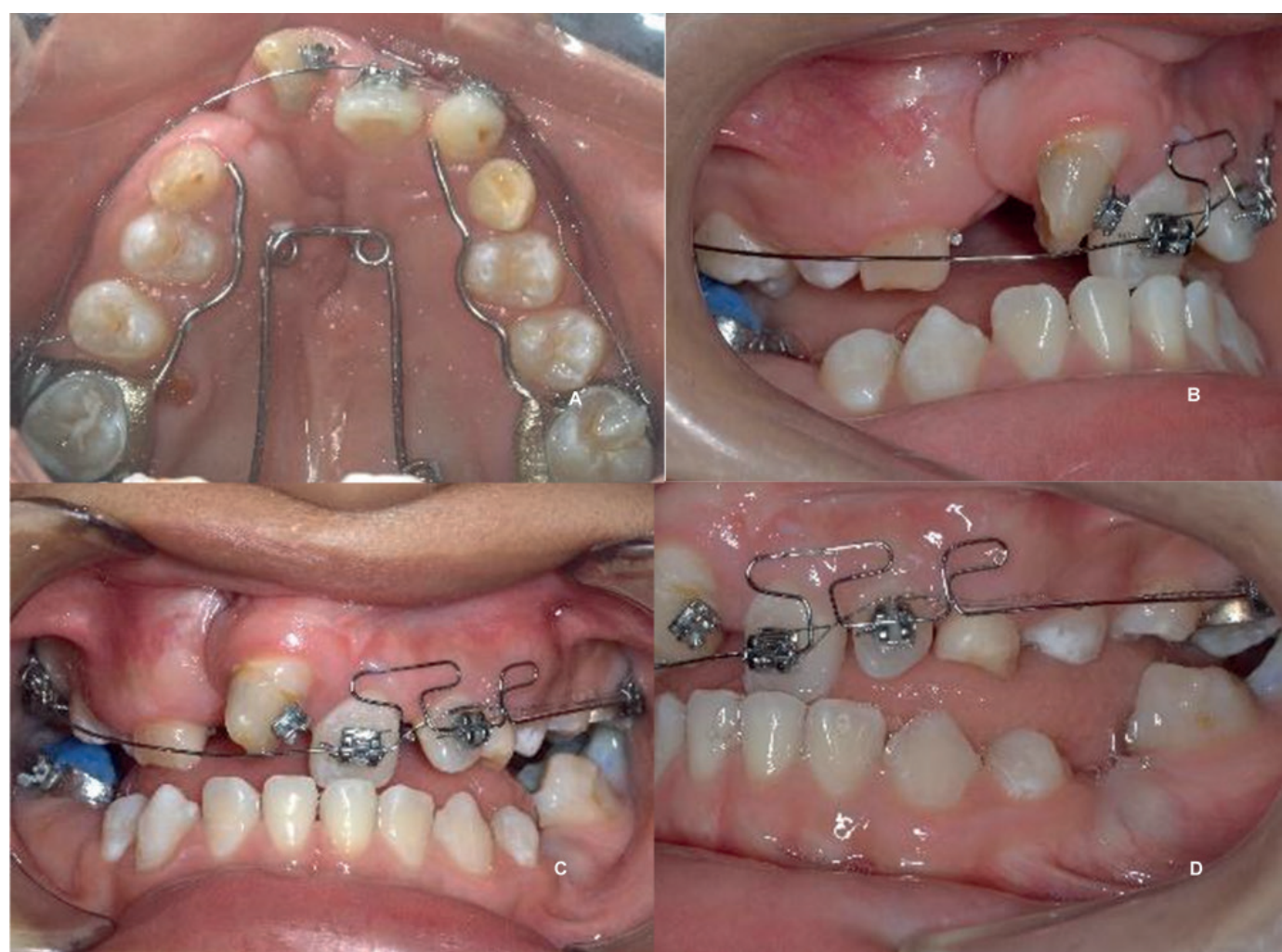

Figura 4. Fotografías de control del caso 1 a los 11 meses. A. Fotografía oclusal. B. Fotografía lateral derecha. C. Fotografía frontal. D. Fotografía lateral izquierda 


\section{Caso 2}

EOM, adolescente de 16 años acude a la Clínica de Posgrado de la Facultad de Odontología porque no le gusta la posición de sus dientes, tiene secuela de fisura labio palatina bilateral, ha tenido intervención del labio a los 3 meses de edad, cirugía del paladar a la edad de un año, sin injerto alveolar.

A la evaluación extraoral es un paciente mesofacial, simétrico y de constitución media, tercio inferior aumentado, perfil recto, presenta el ángulo mentolabial y nasolabial disminuidos, y el mentón retruído; se puede evidenciar también la cicatriz de la cirugía labial (Figura 5).
En las fotografías intraorales se evidencian arcadas parabólicas, la premaxila está separada del resto del maxilar, se evidencia la cicatriz de la cirugía del paladar, tiene mordida cruzada en premolares y canino, la distancia intermolar superior es de $42 \mathrm{~mm}$ y la intercanica es de $36 \mathrm{~mm}$, lo que evidencia el problema transversal en el sector anterior, la sobremordida es de $200 \%$, la relación molar es Clase I bilateral, la línea media superior esta $4 \mathrm{~mm}$ desviada a la izquierda y la inferior está $2 \mathrm{~mm}$ desviada a la derecha con respecto a la línea media facial (Figura 6).

En la radiografía panorámica podemos observar la presencia de un diente supernumerario entre las piezas $13 \mathrm{y}$
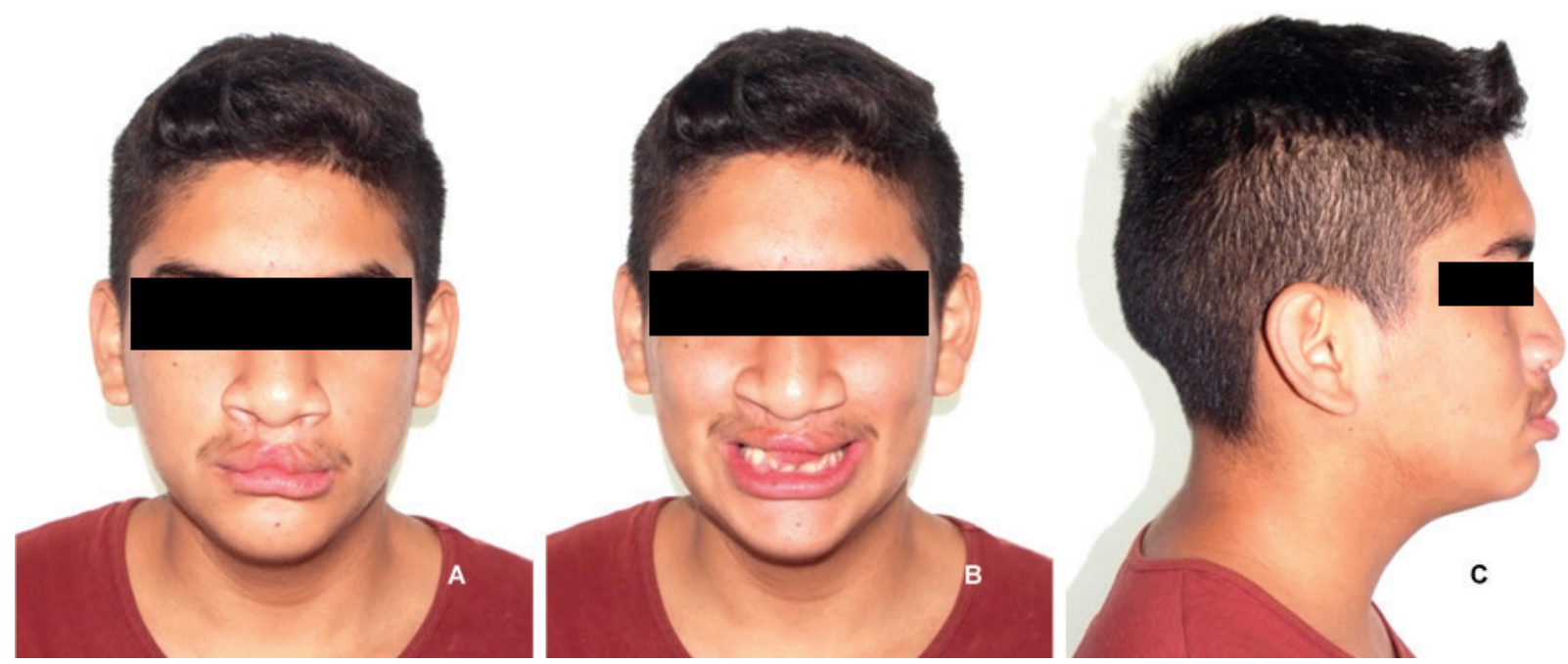

Figura 5. Fotografías extraorales iniciales del caso 2. A. Fotografía frontal. B. Fotografía de sonrisa. C. Fotografía de perfil

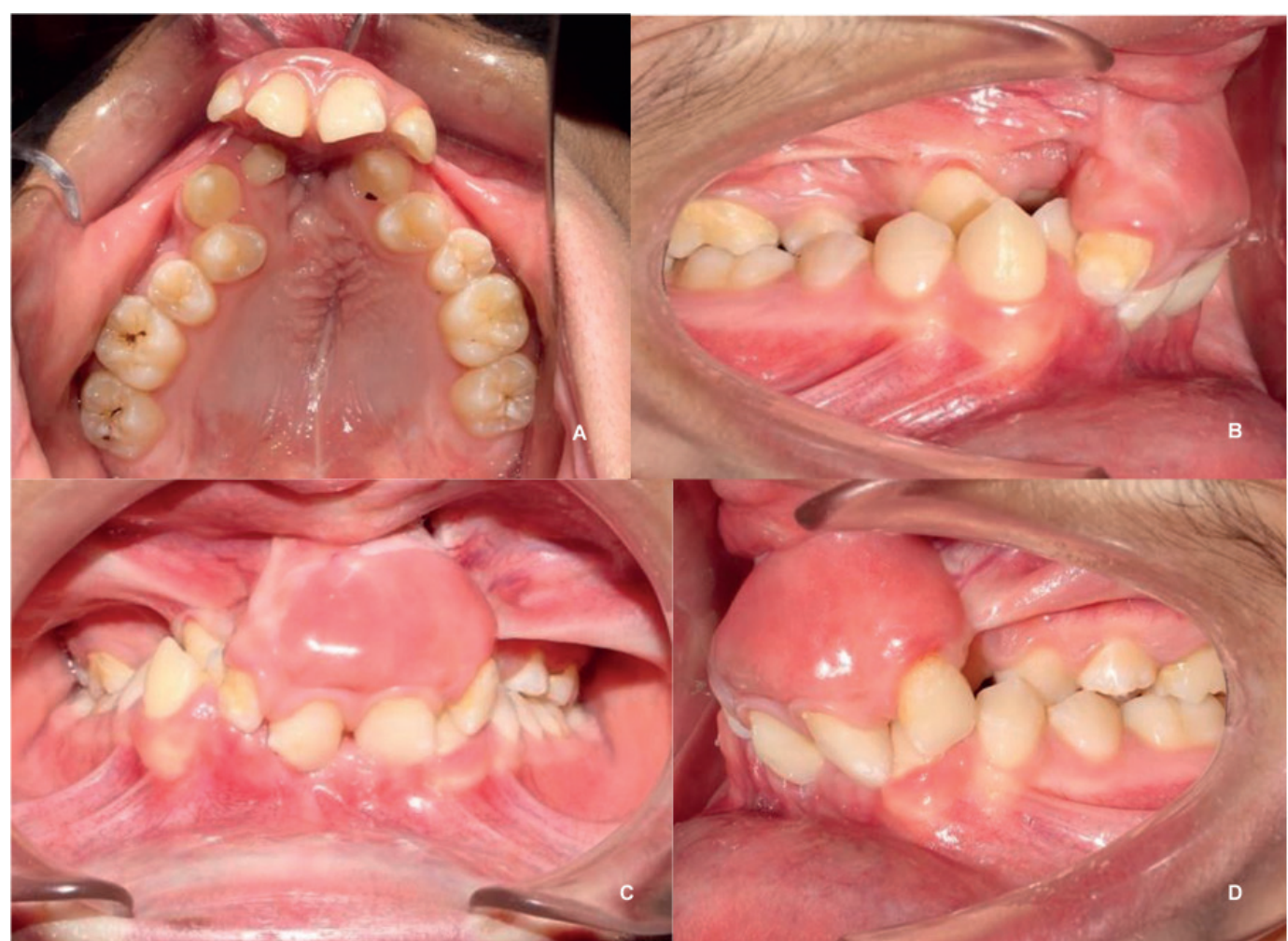

Figura 6. Fotografías intraorales iniciales del caso 2. A. Fotografía oclusal. B. Fotografía lateral derecha. C. Fotografía frontal. D. Fotografía lateral izquierda 
12; aún se evidencia la fisura a nivel óseo a distal de las piezas 12, 22. En la radiografía lateral podemos notar la relación esquelética Clase II con incisivos superior e inferior palatinizados y un perfil óseo recto (Figura 7).

Antes de iniciar el tratamiento de ortodoncia se realizó una interconsulta con el periodoncista, con el especialista de cirugía bucomaxilofacial y con un cirujano plástico; luego se procedió a la extracción del diente supernumerario.
Como plan de tratamiento ortodóntico se optó por la instalación de un expansor quadhelix en la arcada superior para manejar el plano transversal activándose cada dos meses hasta lograr la distancia intermolar de $54 \mathrm{~mm}$ y la intercanina de $38 \mathrm{~mm}$ (Figura 8). La instalación del quadhelix y los brackets se realizó al mismo tiempo, posteriormente en la arcada superior se usó un arco utilitario para producir intrusión en los incisivos y corregir la sobremordida, lo que permitió seguir con la secuencia

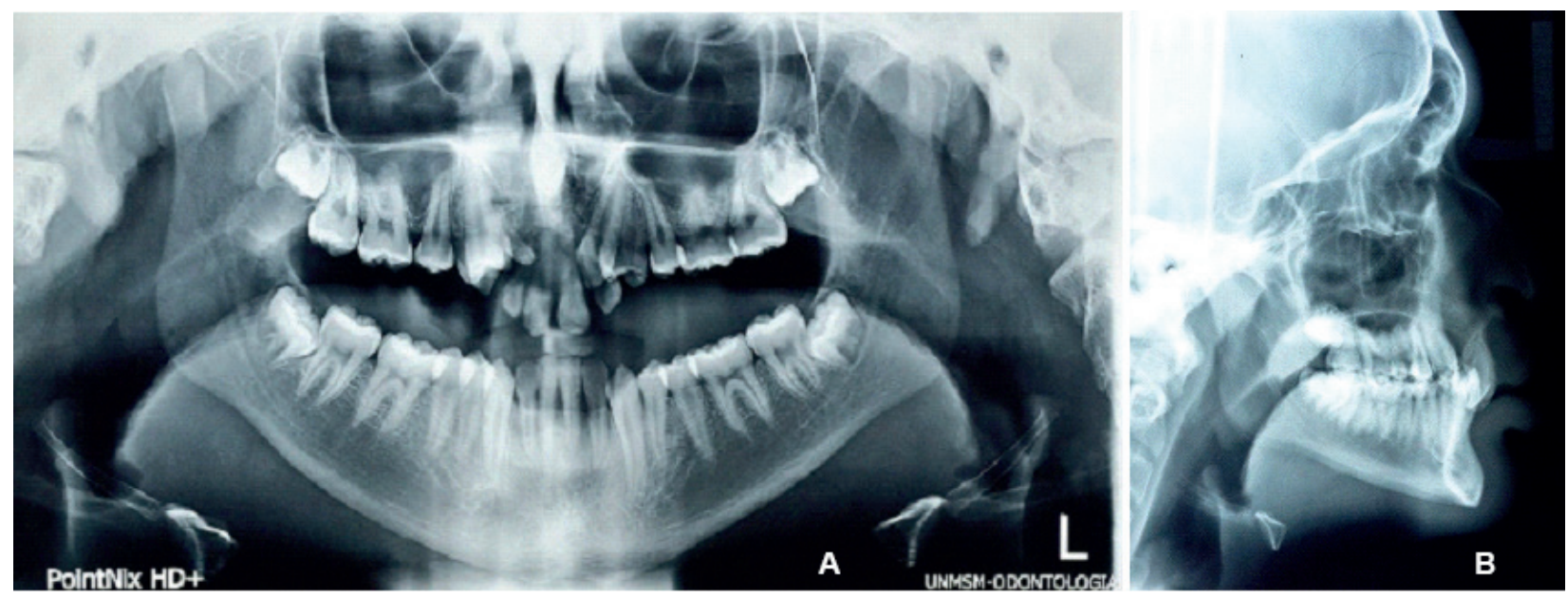

Figura 7. Exámenes auxiliares iniciales del caso 2. A. Radiografía panorámica. B. Radiografía cefalométrica

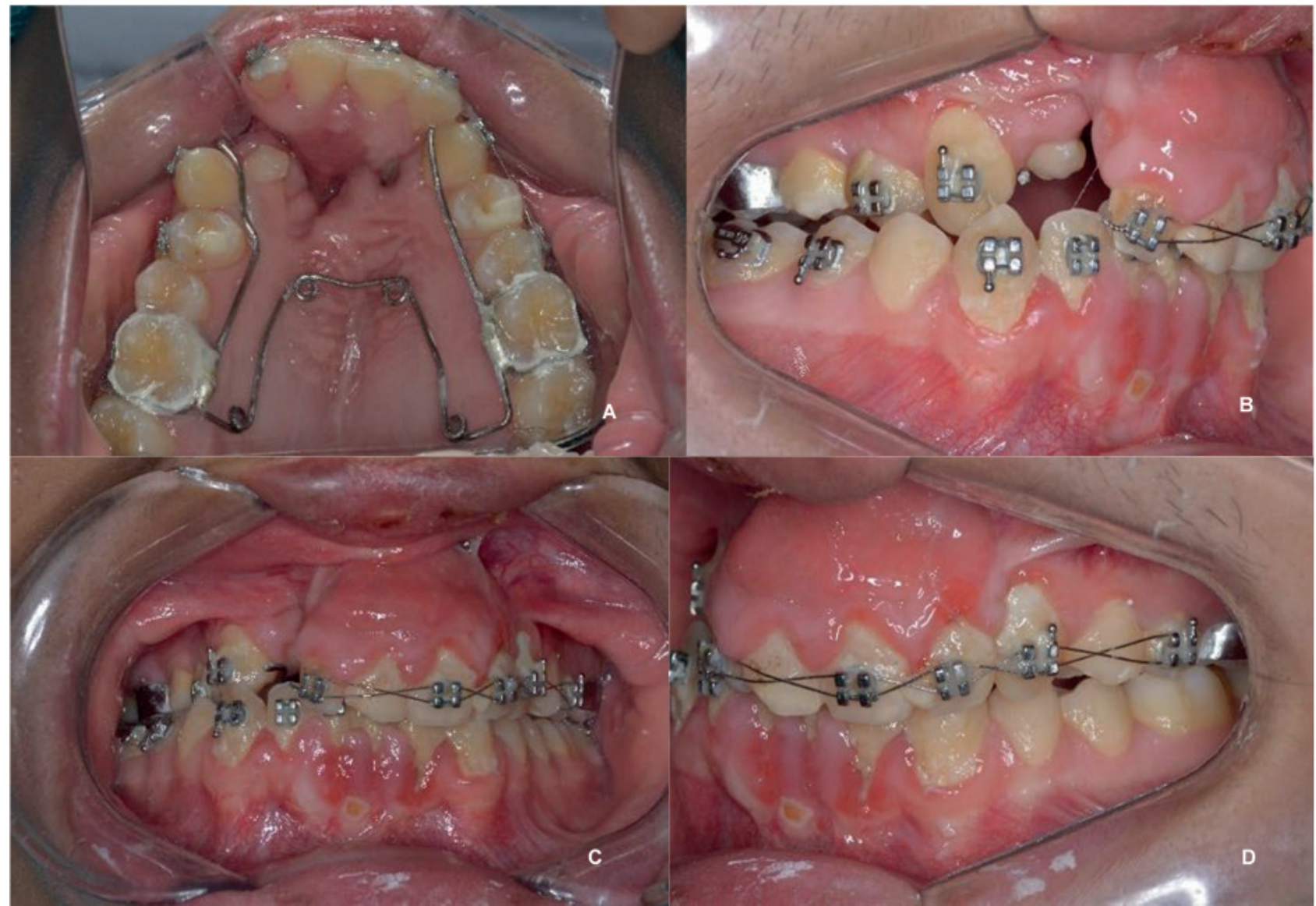

Figura 8. Fotografías de control del caso 2 a los 3 años. A. Fotografía oclusal. B. Fotografía lateral derecha. C. Fotografía frontal. D. Fotografía lateral izquierda 
de arcos para conseguir el alineamiento y nivelación de ambas arcadas. El paciente actualmente se encuentra en tratamiento y aún no se ha realizado el injerto alveolar.

\section{Caso 3}

Paciente masculino de 12 años de edad, se presenta con motivo de consulta de "arreglar sus dientes". Como antecedentes, refiere cirugía de paladar a los 9 meses de edad y de labio a los 3 años de edad, atención fonoaudiológica desde los 12 meses hasta los 5 años de edad.

Al examen extraoral, presenta un patrón mesofacial, con desviación nasal, perfil recto con protrusión de labio inferior, se evidencia la cicatriz de la cirugía labial (Figura 9).

En el examen intraoral, el arco superior presenta forma triangular y apiñamiento dentario severo con múltiples giroversiones, se evidencia un diente supernumerario a nivel del incisivo lateral superior derecho, transposición del canino izquierdo con primer premolar y, canteo. El arco inferior presenta forma cuadrada y apińamiento dentario leve. Las relaciones molares derecha e izquierda son Clase I y Clase II respectivamente. Además, presenta mordida cruzada anterior y posterior, desviaciones de líneas medias superior e inferior, la distancia intercanina superior no es registrable, la distancia interpremolar superior de $25 \mathrm{~mm}$ y la distancia intermolar superior de $48 \mathrm{~mm}$ (Figura 10).

La radiografía panorámica evidencia la pieza 13 retenida. En la radiografía lateral se puede observar una relación esquelética Clase III hiperdivergente por retrusión maxilar (Figura 11).

Se plantea como tratamiento el abordaje transversal con quadhelix con extensiones hasta incisivos laterales. Se colocaron brackets prescripción MBT en los incisivos centrales superiores y arco 0,012 " SS con multiloop para alinearlos. Luego se colocaron brackets en premolares para incluirlos en el arco continuo 0,012" Niti, y también un arco de Mulligan para ayudar a la expansión. Se continuaron con dos activaciones más del quadhelix, de igual manera, se colocaron resortes $\mathrm{NiTi}$ abiertos para crear espacios para los incisivos laterales y vestibularizar los centrales. Se obtiene un arco parabólico con aumento de la distancia interpremolar e intermolar de $3 \mathrm{~mm}$ y $1,5 \mathrm{~mm}$ respectivamente en un periodo de 3 meses ( $\mathrm{Fi}$ gura 12). El paciente aún no recibe injerto, la expansión realizada tuvo como objetivo la preparación previa para la cirugía de injerto óseo.

\section{Discusión}

El manejo transversal en pacientes con fisura labio palatina debe realizarse con mayor cuidado, con el fin de evitar agravar la fisura como podría ocurrir con el Hyrax ${ }^{11}$. La constricción transversal aumenta con la edad como refieren Gregório et al., ${ }^{14}$; por tanto, en los casos que se presentaron se decidió usar el dispositivo quadhelix porque constituye una buena alternativa que evita la pérdida de hueso a nivel vestibular, no empeora la fisura, evita recidivas y permite una mejor adaptación muscular en comparación con la expansión rápida, como manifiestan Gidwani et al., ${ }^{12}$. El quadhelix a pesar de producir una expansión lenta nos permite aumentar el ancho molar y el ancho canino como refieren Frank \& Engel ${ }^{20}$, así mismo, Holberg et al. ${ }^{10}$ encontraron que se producen efectos esqueléticos mucho más marcados en fisuras bilateral; por lo tanto, el quadhelix constituye una alternativa razonable al uso de los dispositivos convencionales.

Pugliese et al. ${ }^{16}$ concluyeron que si se desean cambios en la forma del arco dental, con mayor aumento intercanino que intermolar, el uso de quadhelix es una buena alternativa, debido a que se puede conseguir una mejora en la forma de arcada como se comprobó en estos tres casos que lograron arcadas más parabólicas.

Li \& Lin ${ }^{21}$ encontraron que la expansión del área canina y premolar fue de más de $2 \mathrm{~mm}$. El aumento fue mayor en la región de la primera premolar, luego en las regiones canina y segunda premolar. En este reporte, el

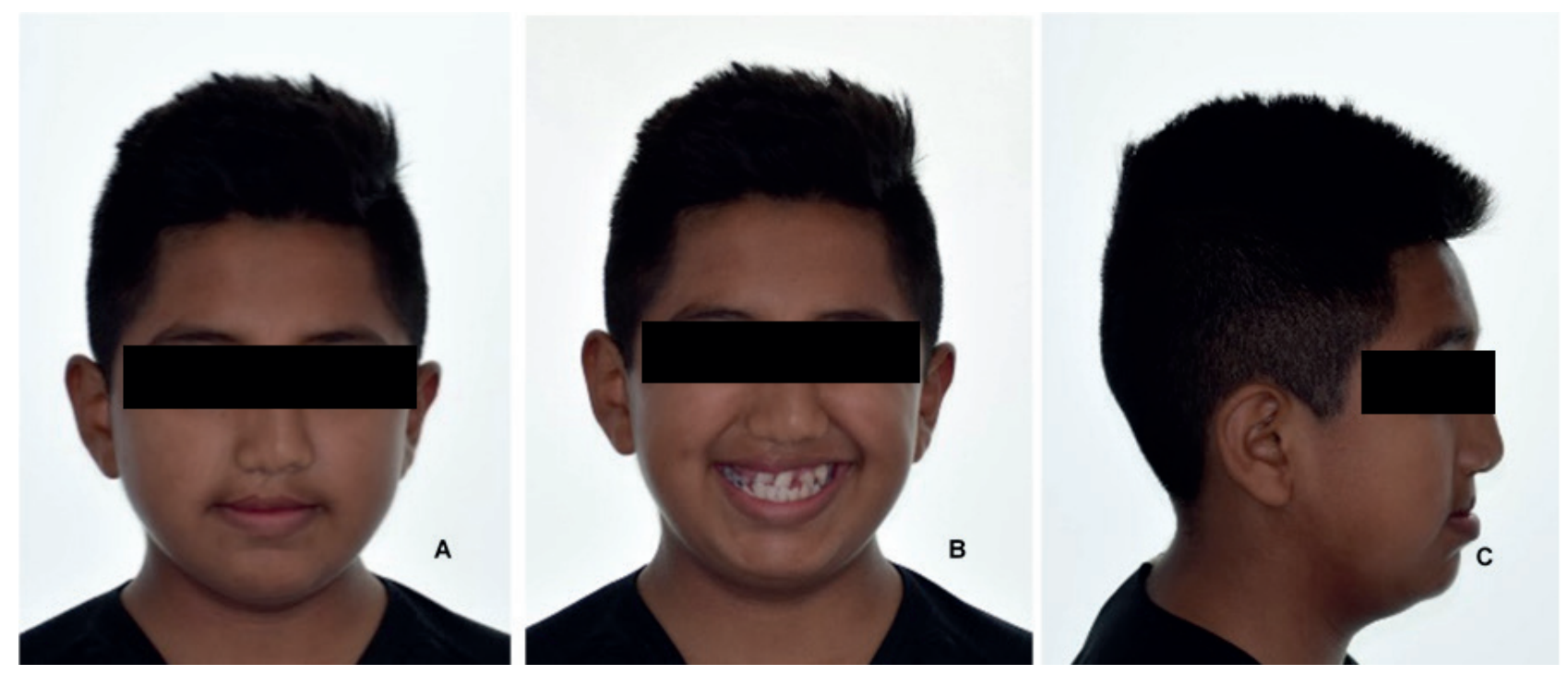

Figura 9. Fotografías extraorales iniciales del caso 3. A. Fotografía frontal. B. Fotografía de sonrisa.

C. Fotografía de perfil 


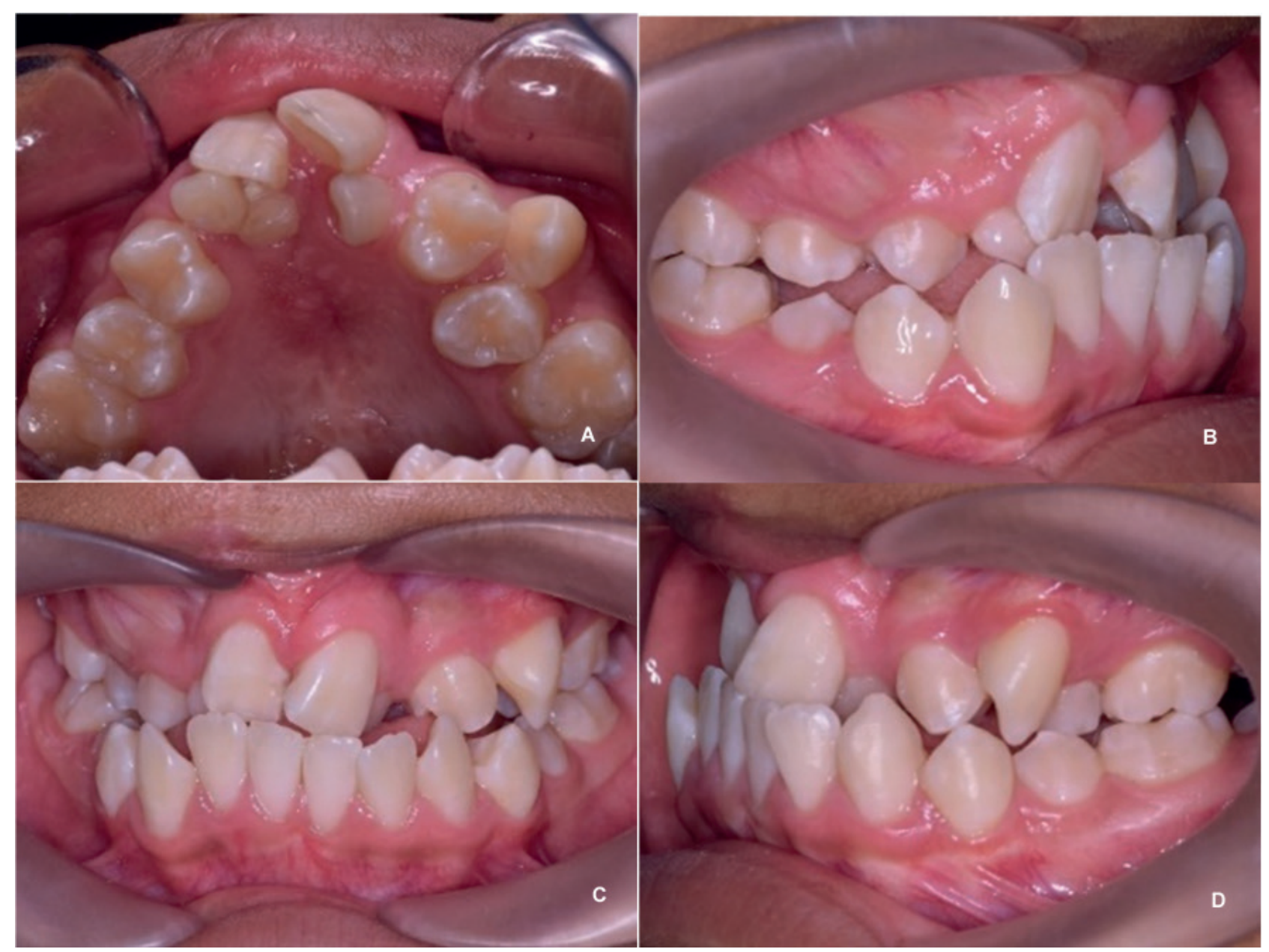

Figura 10. Fotografías intraorales iniciales del caso 3. A. Fotografía oclusal. B. Fotografía lateral derecha. C. Fotografía frontal. D. Fotografía lateral izquierda

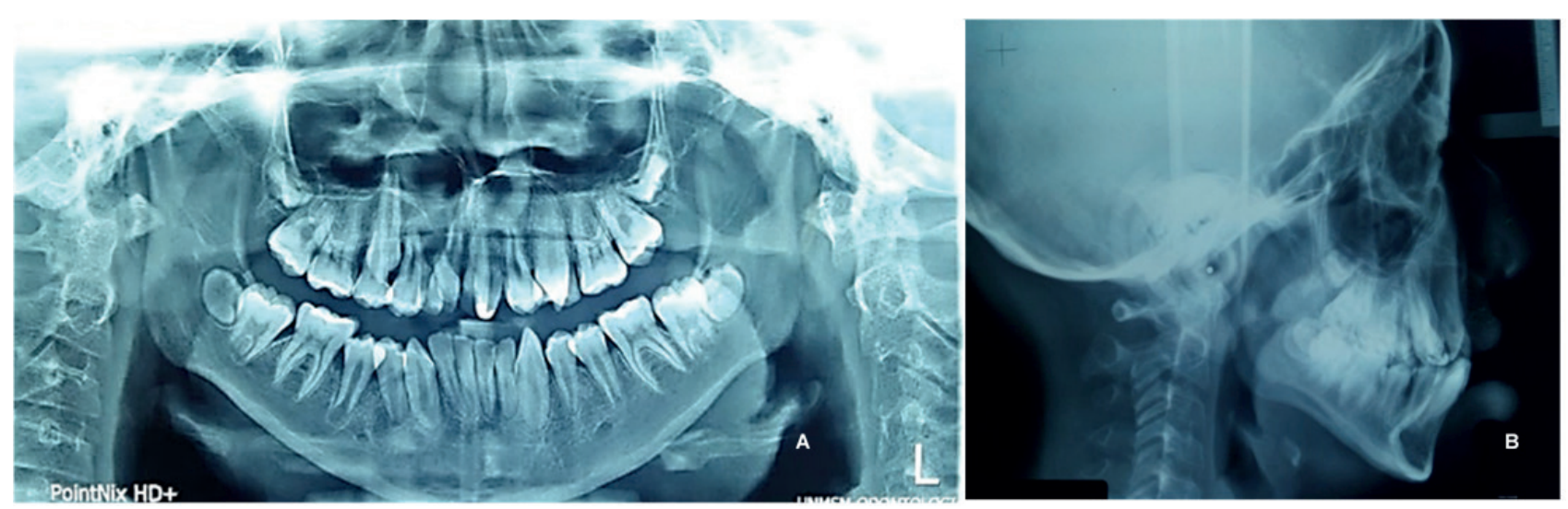

Figura 11. Exámenes auxiliares iniciales del caso 3. A. Radiografía panorámica. B. Radiografía cefalométrica

caso 1 consiguió hasta $6 \mathrm{~mm}$ de aumento en la distancia intercanina.

Bell et al. ${ }^{22}$ encontraron que el aumento promedio entre los caninos maxilares fue de $3,62 \mathrm{~mm}$. La ausencia de la sutura del paladar medio también significa que la expansión preinjerto logra un movimiento segmentario esquelético a expensas de aumentar el ancho de la hendidura. En los casos 1 y 2 se logró 2 y 3 mm de aumento de la distancia intercanina respectivamente. La distancia intermolar en el caso 1 y 2 tuvo mayor aumento comparado al caso 3 debido a la necesidad de expansión anterior que requería este último. En los dos primeros casos, el aumento de la distancia intermolar fue de mayor consideración (6 mm), coincidiendo con Gidwani 12 que reportó una expansión de hasta 5,7 mm inmediatamente después de retirado el quadhelix. De Meiros ${ }^{17}$ obtuvo resultados similares en el grupo de pacientes con fisura labio palatina tratados con quadhelix $(4 \mathrm{~mm})$. En 


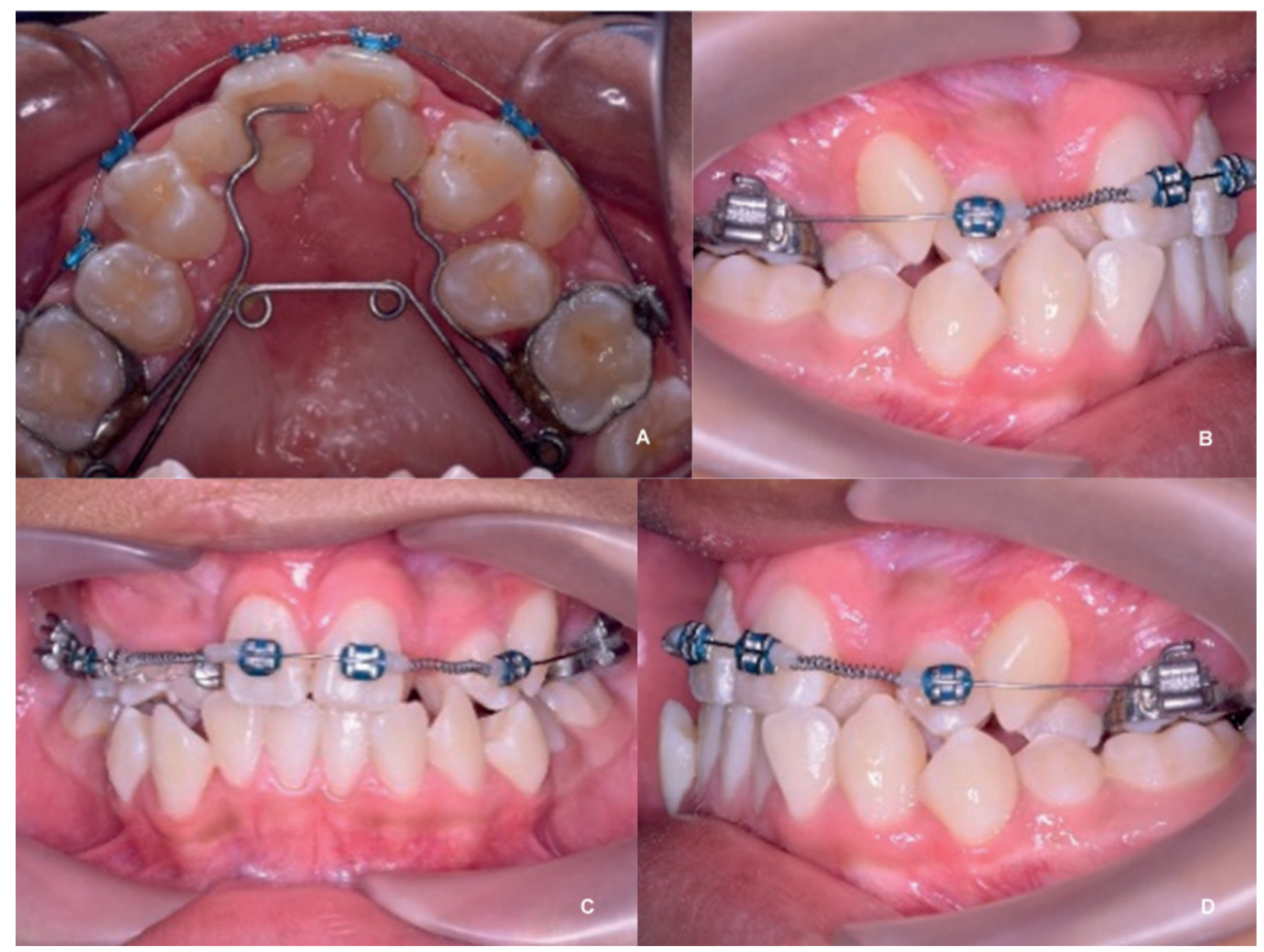

Figura 12. Fotografías de control del caso 3 a los 3 meses. A. Fotografía oclusal. B. Fotografía lateral derecha. C. Fotografía frontal.

D. Fotografía lateral izquierda

cuanto a la cirugía de injerto óseo alveolar, en los tres casos se planificó realizarlo luego de la expansión maxilar, cuando se haya conseguido una mejor forma de arco como refiere Pugliese et al ${ }^{16}$.

En los pacientes con secuela de fisura labio-alveolo-palatina por la deformidad anatómica del hueso maxilar, se puede esperar que el mecanismo de expansión sea diferente, ante esto, el quadhelix representa una alternativa razonable teniendo como ventajas que al ser un dispositivo de expansión lenta, produce menos resistencia tisular alrededor de las estructuras circunmaxilares y mejora la formación de hueso en la sutura intermaxilar; de igual manera al tener mayores efectos dentoalveolares, no produce pérdida ósea a nivel vestibular; adicionalmente presenta una mejor adaptación muscular y bajas probabilidades de recidiva. Por tanto, el quadhelix constituye una alternativa razonable al uso de los dispositivos convencionales.

\section{Referencias bibliográficas}

1. Garmendía GG, Garmendía AM, Morales DV. Propuesta de una metodología de tratamiento en la atención multidisciplinaria del paciente fisurado labio-alveolo-palatino. Rev Cubana Estomatol. 2010;47(2):143-56.
2. Clemente VG, Torres JL-G, Gago AM, Sánchez IN, Fernández LA. Protocolo ortopédico-ortodóncico de actuación en pacientes con fisura labio-alveolar y palatina. Odontol Pediatr. 2017;25(3):173-80.

3. Figueiredo DSF, Bartolomeo FUC, Romualdo CR, Palomo JM, Horta MCR, Andrade I, et al. Dentoskeletal effects of 3 maxillary expanders in patients with clefts: A cone-beam computed tomography study. Am J Orthod Dentofacial Orthop. 2014;146(1):73-81.

4. Garib D, Lauris RDCMC, Calil LR, Alves ACDM, Janson G, De Almeida AM, et al. Dentoskeletal outcomes of a rapid maxillary expander with differential opening in patients with bilateral cleft lip and palate: A prospective clinical trial. Am J Orthod Dentofacial Orthop. 2016;150(4):564-74.

5. de Almeida MR, Marçal ASB, Fernandes TMF, Vasconcelos JB, de Almeida RR, Nanda R. A comparative study of the effect of the intrusion arch and straight wire mechanics on incisor root resorption: A randomized, controlled trial. Angle Orthod. 2018;88(1):20-6.

6. Aizenbud D, Ciceu C, Rachmiel A, Hazan-Molina H. Reverse Quad Helix Appliance: Differential Anterior Maxillary Expansion of the Cleft Area Before Bone Grafting. J Craniofac Surg. 2012;23(5):440-3.

7. López-Palacio A, María Cerón Zapata A, Enrique Cano Acosta A. Rehabilitación temprana de los maxilares en pacientes con 
labio y paladar hendido bilateral utilizando un dispositivo ortopédico dinámico intraoral, cinta adhesiva labial y gingivoperiosteoplastia: estudio piloto experimental. Rev Fac Odontol Univ Antioq. 2009;20(2):138-48.

8. Wang D, Cheng L, Wang C, Qian Y, Pan X. Biomechanical analysis of rapid maxillary expansion in the UCLP patient. Med Eng Phys. 2009;31(3):409-17.

9. Gautam P, Zhao L, Patel P. Biomechanical response of the maxillofacial skeleton to transpalatal orthopedic force in a unilateral palatal cleft. Angle Orthod. 2011;81(3):503-9.

10. Holberg C, Holberg N, Schwenzer K, Wichelhaus A, Rudzki-Janson I. Biomechanical Analysis of Maxillary Expansion in CLP Patients. Angle Orthod. 2007;77(2):280-7.

11. Vasant M, Menon S, Kannan S. Maxillary Expansion in Cleft Lip and Palate using Quad Helix and Rapid Palatal Expansion Screw. Med J Armed Forces India. 2009;65(2):150-3.

12. Gidwani KV, Bendgude VD, Kokkali VV, Mehta V. Comparison of effectiveness of quad helix appliance with other slow maxillary expanders in children with posterior crossbite: A systematic review. J Indian Soc Pedod Prev Dent. 2018;36(3):225-33.

13. Gregório L, de Medeiros Alves AC, de Almeida AM, Naveda R, Janson G, Garib D. Cephalometric evaluation of rapid and slow maxillary expansion in patients with BCLP: Secondary data analysis from a randomized clinical trial. Angle Orthod. 2019;89(4):583-9.

14. Ricketts RM. Growth prediction: Part 2. J Clin Orthod. 1975;9:340-362.

15. Bench RW. The quad helix appliance. Semin Orthod. 1998;4(4):231-7.
16. Pugliese F, Martin Palomo J, Resti Calil L, Alves A, Lauris J, Garib D. Dental arch size and shape after maxillary expansion in bilateral complete cleft palate: A comparison of three expander designs. Angle Orthod. 2019; DOI: 10.2319/02021974.1. [Epub ahead of print]

17. de Medeiros Alves AC, Garib DG, Janson G, de Almeida AM, Calil LR. Analysis of the dentoalveolar effects of slow and rapid maxillary expansion in complete bilateral cleft lip and palate patients: a randomized clinical trial. Clin Oral Invest. 2016;20(7):1837-47.

18. Kilpatrick N. Management of cleft lip and palate. En: Handbook of Pediatric Dentistry [Internet]. Elsevier; 2013 [citado 1 de septiembre de 2019]. p. 447-461. Disponible en: https:// linkinghub.elsevier.com/retrieve/pii/B9780723436959000158

19. Uzel A, Benlidayı ME, Kürkçü M, Kesiktaş E. The effects of maxillary expansion on late alveolar bone grafting in patients with unilateral cleft lip and palate. J Oral Maxillofac Surg. 2019;77(3):607-14.

20. Frank SW, Engel GA. The effects of maxillary quad-helix appliance expansion on cephalometric measurements in growing orthodontic patients. Am J Orthod. 1982;81(5):378-89.

21. Li W, Lin J. Dental arch width stability after Quadhelix and edgewise treatment in complete unilateral cleft lip and palate. Angle Orthod. 2007;77(6):1067-72.

22. Bell RA, LeCompte EJ. The effects of maxillary expansion using a quad-helix appliance during the deciduous and mixed dentitions. Am J Orthod. 1981;79(2):152-61. 